OPEN ACCESS

Edited by:

Kimberley Cathryn Snowden,

Plant and Food Research,

New Zealand

Reviewed by:

Krystyna Oracz,

Warsaw University of Life

Sciences-SGGW, Poland

Mark Waters,

The University of Western Australia,

Australia

${ }^{*}$ Correspondence:

Wenyu Yang

mssiyangwy@sicau.edu.cn

Kai Shu

kshu@sicau.edu.cn

tThese authors have contributed equally to this work.

Specialty section:

This article was submitted to Plant Evolution and Development,

a section of the journal

Frontiers in Plant Science

Received: 02 September 2016 Accepted: 19 December 2016

Published: 24 January 2017

Citation:

Meng Y, Shuai H, Luo X, Chen F, Zhou W, Yang $W$ and Shu $K$ (2017)

Karrikins: Regulators Involved in Phytohormone Signaling Networks during Seed Germination and Seedling Development.

Front. Plant Sci. 7:2021.

doi: 10.3389/fpls.2016.02021

\section{Karrikins: Regulators Involved in Phytohormone Signaling Networks during Seed Germination and Seedling Development}

\author{
Yongjie Meng ${ }^{\dagger}$, Haiwei Shuait, Xiaofeng Luo, Feng Chen, Wenguan Zhou, Wenyu Yang* \\ and Kai Shu* \\ Key Laboratory of Crop Ecophysiology and Farming System in Southwest China (Ministry of Agriculture), Sichuan \\ Engineering Research Center for Crop Strip Intercropping System, Institute of Ecological Agriculture, Sichuan Agricultural \\ University, Chengdu, China
}

Seed germination and early seedling establishment are critical stages during a plant's life cycle. These stages are precisely regulated by multiple internal factors, including phytohormones and environmental cues such as light. As a family of small molecules discovered in wildfire smoke, karrikins (KARs) play a key role in various biological processes, including seed dormancy release, germination regulation, and seedling establishment. KARs show a high similarity with strigolactone (SL) in both chemical structure and signaling transduction pathways. Current evidence shows that KARs may regulate seed germination by mediating the biosynthesis and/or signaling transduction of abscisic acid (ABA), gibberellin (GA) and auxin [indoleacetic acid (IAA)]. Interestingly, KARs regulate seed germination differently in different species. Furthermore, the promotion effect on seedling establishment implies that KARs have a great potential application in alleviating shade avoidance response, which attracts more and more attention in plant molecular biology. In these processes, KARs may have complicated interactions with phytohormones, especially with IAA. In this updated review, we summarize the current understanding of the relationship between KARs and SL in the chemical structure, signaling pathway and the regulation of plant growth and development. Further, the crosstalk between KARs and phytohormones in regulating seed germination and seedling development and that between KARs and IAA during shade responses are discussed. Finally, future challenges and research directions for the KAR research field are suggested.

Keywords: karrikins, ABA, GA, IAA, germination, photomorphogenesis

\section{INTRODUCTION}

Most angiosperm plants start a new stage of growth and development with seed germination. Although seed dormancy prevents germination, it is a very important approach for plant survival, especially under unfavorable conditions. Seeds can define whether the environmental conditions are appropriate for germination (Finch-Savage and Leubner-Metzger, 2006; Shu et al., 2015; Oracz and Stawska, 2016). For the dormant seeds, a series of environmental and endogenous signals cooccur to break seed dormancy, and then induce germination. Seedling establishment is another key 
stage of plant life cycle, which follows closely after germination. It is believed that well-developed seedlings result in well-developed plants (Finch-Savage et al., 2010; Imran et al., 2013).

Phytohormones play a dominant role in regulating seed germination and seedling establishment. Gibberellins (GA) can break seed dormancy and induce germination (Yamauchi et al., 2004), while abscisic acid (ABA) can promote seed dormancy and delay germination (Ali-Rachedi et al., 2004). Auxin [indoleacetic acid (IAA)] is also involved in regulating seed dormancy (Liu et al., 2013). Furthermore, IAA has been demonstrated to be an important regulator in the plant shade avoidance syndrome that adversely affects seedling development and crop yield (Casal, 2013a; Gommers et al., 2013; Procko et al., 2014). In addition to phytohormones, other chemical compounds have the ability to regulate plant growth and development, such as nitrogen oxide and reactive oxygen species (ROS), both of which have been demonstrated to regulate seed dormancy and germination (Bethke et al., 2006; Oracz et al., 2007, 2009; Oracz and Karpiński, 2016).

In 2004, chemists purified 3-methyl-2H-furo [2, 3-c] pyran2-one from the smoke of burning plant material (Flematti et al., 2004). Subsequently, several analogs to 3-methyl-2Hfuro $[2,3-c]$ pyran-2-one were found and collectively named as karrikins (Flematti et al., 2007; Dixon et al., 2009). Subsequent studies revealed that KARs have significant biological activities in promoting germination and seedling establishment of model plant Arabidopsis (Waters and Smith, 2013; Flematti et al., 2015). KARs may regulate seed germination and shade responses by interacting with endogenous phytohormones signaling networks. In this review article, the relationship between KARs and SL is summarized, and then we discuss the mechanisms through which KARs interact with different phytohormones, and the crosstalk among KARs, ABA, GA, and auxin in the processes of germination and early seedling establishment. Finally, the challenges and research directions in the following study of KARs research field are suggested.

\section{THE RELATIONSHIP BETWEEN KARS AND SL}

So far, six different isoforms of KARs family are documented, $\mathrm{KAR}_{1}-\mathrm{KAR}_{6}$; and all of which contain a five-membered butenolide ring and a six-membered pyran ring (Dixon et al., 2009; Flematti et al., 2009; Waters et al., 2014). The primary difference among KARs family members is the number and location of methyl group(s) (Flematti et al., 2009). Interestingly, the butenolide moiety of KARs has high similarities with the D-ring of SL, a compound which is synthesized and exuded from roots, and also triggers the germination of parasitic weeds (Yoneyama et al., 2007; Dor et al., 2010; Waters et al., 2012).

Due to the significant promotion effect of KARs on seed germination of some species, the detailed mechanisms of KARs signaling has always been one of the most written topics in this field. KARRIKIN INSENSITIVE2 (KAI2) is the receptor in the signaling pathway of KARs (Figure 1) (Waters et al., 2013). When bound by KARs, KAI2 undergoes conformational

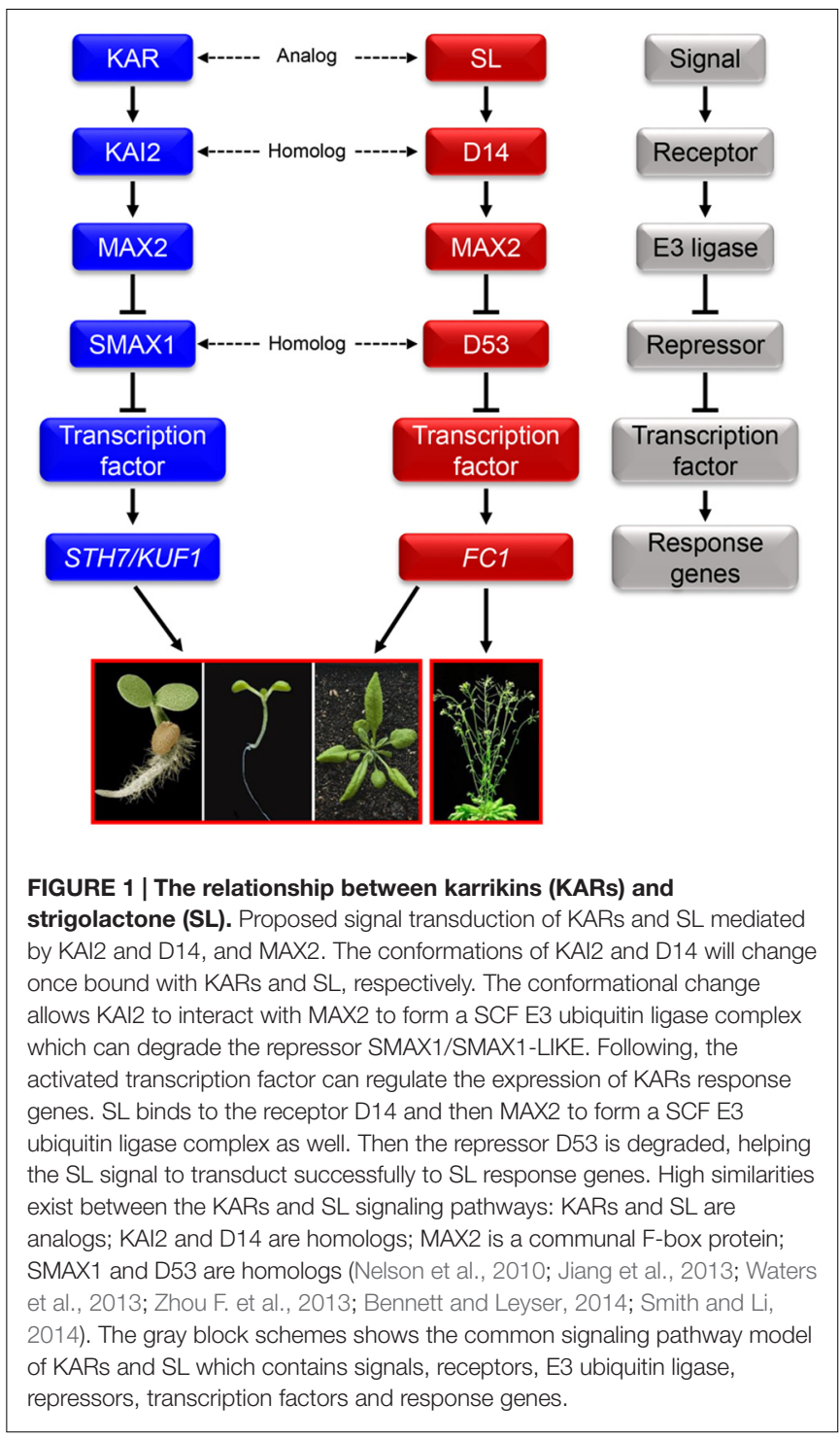

changes (Guo et al., 2013; Zhao et al., 2013). Subsequently, KARs and KAI2 might form a SCF E3 ligase complex with MORE AXILLARY GROWTH2 (MAX2) (Waters et al., 2012, 2013; Kagiyama et al., 2013). The SCF complex can then promote the degradation of SMAX1 which is a repressor in KARs signaling pathway (Stanga et al., 2013). Further, other repressors of the KARs signaling pathway have been documented and named as SMAX1-LIKEs (Stanga et al., 2013). It is noted that the various repressors involved in its signaling pathway lead to the diversiform biological functions of KARs (Smith and Li, 2014).

As well as the similarities in chemical structures between KARs and SL, many components of these two signaling pathways are analogs or homologs (Figure 1) (Flematti et al., 2015; Morffy et al., 2016). Firstly, both signaling pathways are composed of receptors, E3 ligases and signal repressors; secondly, the receptor of SL signal, AtD14, is a homolog of KAI2 which is the receptor of KARs (Kagiyama et al., 2013; Waters et al., 2013); thirdly, the repressor of SL signaling pathway, D53, is also a homolog of 
SMAX1, the repressor in KARs signaling transduction pathway (Jiang et al., 2013; Zhou F. et al., 2013).

The similar chemical structures and signaling pathways of KARs and SL suggest common biological functions. Extensive studies reveal that both KARs and SL can promote seed germination and inhibit hypocotyl elongation (Nelson et al., 2010; Waters and Smith, 2013). However, the delicate distinctions between KARs and SL can result in some differences in other biological functions. For example, AtD14 cannot replace KAI2 during the processes of seed germination and seedling development; while KAI2 cannot take the place of AtD14 to regulate branch formation (Waters et al., 2015). Furthermore, GR24, a synthetic analog of SL, could not promote the expansion of cotyledons, while KARs could (Nelson et al., 2010); and GR24 could repress shoot branching, but KARs could not (Fukui et al., 2011; Nelson et al., 2011; Boyer et al., 2012). These striking differences in bioactivities of the two structurally similar butenolide compounds imply two distinct response systems in plants (Scaffidi et al., 2013), although the detailed components and precise mechanisms still need further dissection.

The signaling pathway mode of KARs and SL is very important and generally occurs in phytohormones signaling transduction, such as GA, IAA, and salicylic acid (Schwechheimer, 2008; Xu et al., 2010; Sun, 2011; Van der Does et al., 2013). If the signal is weak or absent, the receptors remain dormant as well as the E3 ubiquitin ligase, and subsequently the repressors repress the transcription of response genes. However, in the presence of signals, the activated E3 mediates the degradation of repressors and release the expression of response genes to regulate plant growth and development.

\section{KARS REGULATE GERMINABILITY OF SEEDS BY INTERACTING WITH PHYTOHORMONES IN Arabidopsis}

Seed dormancy and germination are not only important to plants but also to human beings, since germination rate is one of the main determinants that affects production in agriculture systems. KARs induce seed germination under weak light conditions by enhancing the response of seeds to light (Drewes et al., 1995; Nelson et al., 2010), whereas the acceleration effect of KARs on germination disappears in dark conditions (Nelson et al., 2010). However, fresh Arabidopsis seeds are insensitive to KARs, but the seeds become sensitive to KAR treatment after the after-ripening treatment (Waters et al., 2014). Further, the acceleration effect of KARs on seed germination also depends on Arabidopsis ecotype and depth of seed dormancy (Nelson et al., 2009).

During the process of germination, subtle changes in environmental conditions can be sensed by seeds and these cues can further affect internal signals such as phytohormones signaling networks. Numerous studies demonstrated that ABA and GA antagonistically regulate seed germination (Daws et al., 2007). ABA induced seed dormancy and inhibited germination, while GA had converse effects on those processes (Figure 2) (Xi et al., 2010). Consequently, the ratio of ABA/GA had a decisive and critical effect on the process of seed germination

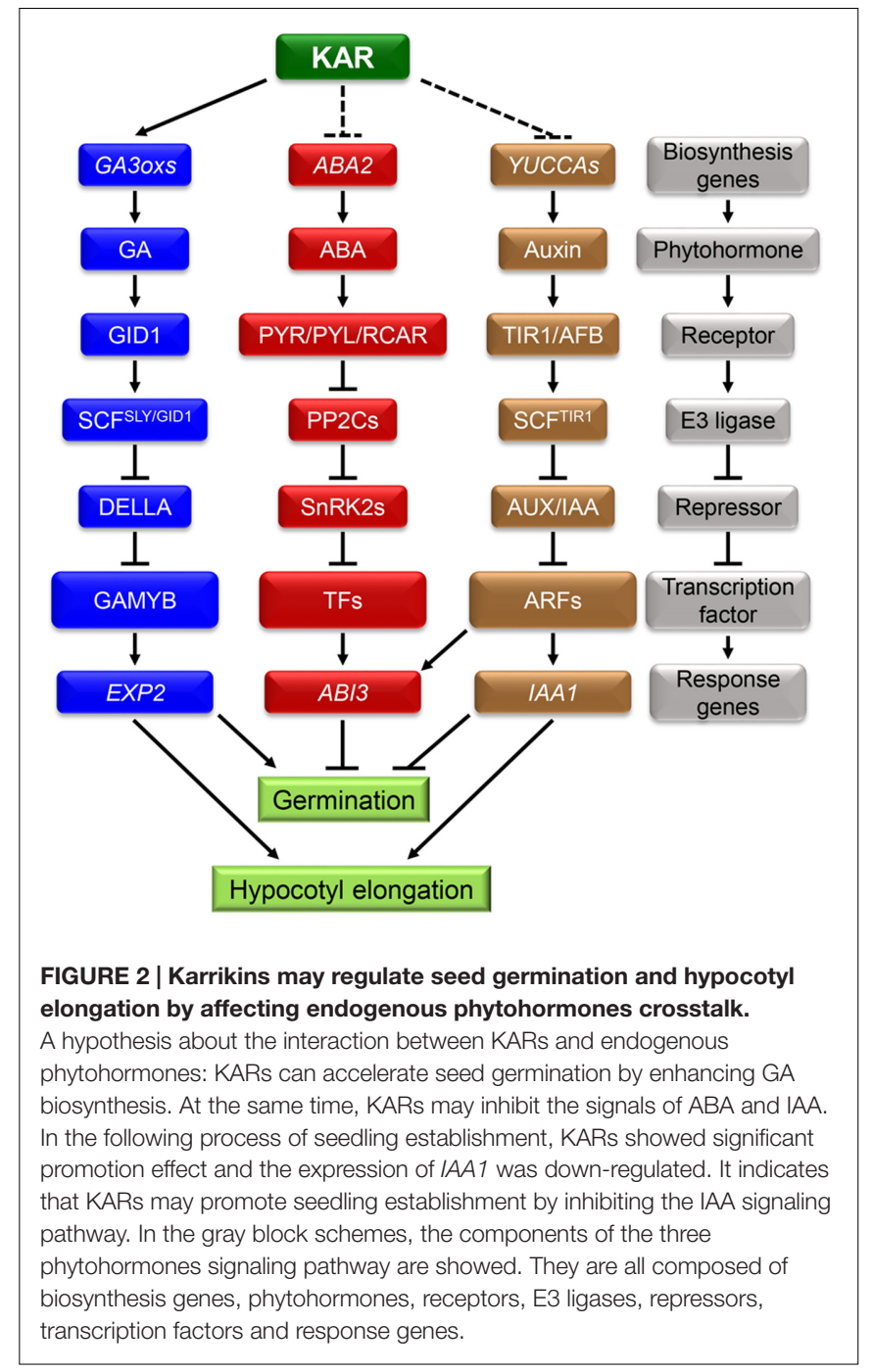

(Seo et al., 2006; Shu et al., 2013; Meng et al., 2016). In conclusion, the dynamic balance between ABA and GA has a unique role in regulating seed dormancy and germination (Finkelstein et al., 2008). Recent studies showed that IAA can also regulate seed dormancy and germination (Figure 2). Exogenous IAA effectively inhibited the pre-harvested sprouting of wheat spikelets (Ramaih et al., 2003). Furthermore, the process of germination was strongly inhibited in the transgenic plants iaaM-OX which possess higher levels of IAA in seeds (Cheng et al., 2006). On the contrary, the mutation in IAA biosynthesis genes YUCCAs led to lowering seed dormancy level (Liu et al., 2013). All of this evidence indicates that IAA has an important role in promoting seed dormancy and inhibiting germination.

Since ABA, GA and IAA are all involved in regulating seed dormancy and germination, a hypothesis that there is an interaction between KARs and those phytohormones needs to be further investigated. A former study showed that ABA removes the acceleration effect of KARs on germination and KARs need the biosynthesis of GA to promote seed germination (Nelson et al., 2009). In addition, KARs treatment promoted 
the expression of GA biosynthesis genes GA3ox1 and GA3ox2 (Nelson et al., 2010), but no transcription evidence is investigated about that of ABA thus far.

Indoleacetic acid regulates seed dormancy and germination by mediating the signal of ABA. AXR2 and AXR3 are transcriptional repressors of IAA signaling pathway (Sabatini et al., 1999; Nagpal et al., 2000). The seeds of axr2-1 and axr3-1, which have a weaker endogenous IAA signal, are insensitive to ABA; but the blockingup of IAA signaling pathway has no effect on the endogenous ABA content (Liu et al., 2013). On the contrary, aba2, the ABA deficient mutant, did not affect seed sensitivity to IAA. These results suggest synergistic effects between IAA and ABA in the process of germination. Subsequent investigations showed that the seeds of abi3-1 mutant germinate normally in the presence of exogenous IAA and ABA (Liu et al., 2013). Furthermore, during the process of seed imbibition, $A B I 3$ transcription remained at a higher level in iaaM-OX transgenic plants, compared to wild type. On the contrary, the $A B I 3$ mRNA content in arf10 arf16 seeds decreased gradually during imbibition (Liu et al., 2013). It indicates that $\mathrm{ABA}$ can regulate seed germination and dormancy in an IAA-dependent manner. Further, ABA can positively regulate seed dormancy by inhibiting GA signaling and working synergistically with IAA; GA and IAA may therefore regulate seed dormancy antagonistically. But the precise mechanism underlying the synergy effect between ABA and IAA, and antagonism between GA and IAA remains elusive so far.

Importantly, KARs suppress the expression of IAA1 which is IAA response genes (Yang et al., 2004; Nelson et al., 2011; Gilkerson et al., 2015). Furthermore, as the analog of KARs, SL could regulate shoot branching by triggering the degradation of PIN1 which determines the polar transportation of IAA (Petrasek et al., 2006; Shinohara et al., 2013). Accordingly, KARs may accelerate seed germination by suppressing the signals of IAA. Whether KAI2 and MAX2 are also involved in the interactions between KARs and phytohormones during seed germination still needs further investigation. Furthermore, both KARs and IAA interact with $\mathrm{ABA}$ during germination, thus whether KARs affect ABA signal by regulating IAA signaling pathway is still unknown.

\section{THE EFFECT OF KARS ON CROP SEED GERMINATION}

Most investigations about the acceleration effect of KARs on seed germination have been focused on the model plant Arabidopsis (Nelson et al., 2010; Waters et al., 2013) and the fire-following species (Keeley and Pizzorno, 1986; Daws et al., 2007). Subsequent investigations revealed that many weed seeds, even some horticultural crop seeds such as lettuce (Lactuca saliva) and tomato (Lycopersicon esculentum) were responsive to KARs (Drewes et al., 1995; Jain et al., 2006; Stevens et al., 2007). Can KARs be applied to regulate seed germination of crops? A recent study has demonstrated that KARs delayed soybean seed germination by enhancing ABA biosynthesis and impairing GA biogenesis (Meng et al., 2016). Surprisingly, KARs only inhibited soybean seed germination under shade conditions, rather than white light and dark conditions, which is completely distinct from the effect of KARs in Arabidopsis. Quantification of phytohormones showed that KARs enhanced ABA biosynthesis while impairing that of GA, and subsequently resulted in the decrease of $\mathrm{GA}_{4} / \mathrm{ABA}$ ratio. The following evidence including transcription patterns of ABA and GA metabolic related genes and inhibitors of ABA biosynthesis was consistent with the phenotype and hormone quantification (Meng et al., 2016). In conclusion, KARs delay soybean seed germination by regulating the ratio of GA/ABA under shaded conditions. Apart from soybean, the germination of other species such as Capsella bursapastoris, Bromus sterilis, and Alopecurus myosuroide could also be inhibited by KARs (Daws et al., 2007), but the detailed mechanisms still need further dissection.

Why do KARs repress seed germination in some species, such as soybean? It is noted that soybean originates in China, a nonMediterranean climate region. It was suggested that the difference of environment may result in different response mechanisms in the evolution history (Meng et al., 2016). Secondly, cultivated soybean is artificially bred. Compared with the wild soybean, some critical genes might encounter deficiency or mutation which would also result in a distinct response mechanism to KARs treatment (Meng et al., 2016).

Though KARs did not show any acceleration effect on seed germination in soybean, KARs may still have applications in agricultural production. For example, treating field soil with KARs may cause "suicidal germination" of agricultural weeds so that the weeds can be eliminated easily (Flematti et al., 2015). Preharvest sprouting of soybean, especially under high temperature and humidity conditions, has an extremely negative impact on seed yield and nutritional quality (Quinhone and Ida, 2015; Shu et al., 2015). Based on the inhibition effect of KARs on soybean seed germination, spraying the KARs solution on mother plants in natural field may decrease pre-harvest sprouting of soybean. In future work, the effect of KARs on seed germination of other crops such as wheat, rice and maize still needs further analysis.

\section{SHADE: POTENTIAL APPLICATION OF KARs}

Seedling development is another critical phase in the plant life cycle (Eastmond et al., 2015). In natural plant community or agricultural system, vegetation canopy decreases the red/far red light ratio and light intensity sensed by lower blades (Franklin, 2008; Casal, 2012). Shade affects almost all stages of growth and development of plants, including seed germination, seedling development and stem elongation (Valladares and Niinemets, 2008; Casal, 2013b). In most cases, the effects of shade are undesirable, including excessive growth and lower resistance to biotic and abiotic stresses (Kobata et al., 2000; Ballaré et al., 2012; Wit et al., 2013). In the face of shade stress, plants have evolved two completely distinct response mechanisms: shade tolerance and shade avoidance (Gommers et al., 2013).

Current studies showed that KARs could enhance the sensitivity of seedlings to light and promote seedling establishment (Waters and Smith, 2013). The hypocotyl was greener in KARs treatment and the chlorophyll content 
was higher; furthermore, the elongation of the hypocotyl was inhibited by KARs; and these promotion effects were independent of the plant genetic background (Nelson et al., 2010). Since KARs are so helpful in the regulation of photomorphogenesis by inducing sensitivity of seedlings to light, it is hypothesized that KARs may be an efficient solution to attenuate plant shade avoidance syndrome.

\section{KARs MAY INTERACT WITH IAA TO ELIMINATE SHADE RESPONSE}

Phytohormones always play an efficient role in regulating the shade response of plants. Numerous studies showed that IAA, GA and brassinolide induce the elongation of hypocotyl by promoting cell elongation (Lilley et al., 2012; Chen et al., 2013; Bernardo-García et al., 2014; Oh et al., 2014). Further, both GA and brassinolide regulate hypocotyl growth in an IAA-dependent manner (Stamm and Kumar, 2010; Chapman et al., 2012; Zhou X.-Y. et al., 2013). Consequently, IAA appears to be the most dominant regulator in shade avoidance response regarding plant hypocotyl elongation.

On one hand, IAA content in the hypocotyl significantly increased under shade conditions; on the other hand, mutants deficient in IAA biosynthesis were insensitive to shade stress (Stone et al., 2008; Tao et al., 2008; Keuskamp et al., 2010; Cole et al., 2011). A further study in Brassica rapa also showed that, the excess IAA was biosynthesized in the cotyledons and transported to the hypocotyl under shade conditions (Procko et al., 2014). In conclusion, shade stress may regulate hypocotyl elongation mainly by promoting the biosynthesis and transportation of IAA. As KARs repressed the expression of IAA1 (Figure 2) (Nelson et al., 2011), thus a hypothesis is proposed: the biosynthesis and transport of IAA in the process of seedling establishment may be inhibited by KARs. But whether KARs promote seedling establishment by inhibiting the IAA signaling pathway still needs more investigation.

As a critical factor in KARs signaling pathway, MAX2 may have important roles in regulating germination and seedling development. max2 mutants showed deep seed dormancy, epinastic leaves and long hypocotyls under white light, red light, far-red light, and blue light conditions (Nelson et al., 2011; Waters et al., 2012; Stanga et al., 2013; Jia et al., 2014). This suggests that $M A X 2$ is a positive regulator of photomorphogenesis (Shen et al., 2007). Consequently, the relationship between $M A X 2$ and the light signaling pathway needs further investigation. As a negative regulator in light signaling pathway, quadruple mutant of PIF (pifq) showed enhanced germination and seedling establishment under both dark and red light conditions (Shin et al., 2009). The seeds of double mutant between pif1 and max 2 showed an intermediate germination rate phenotype. Further, the double mutants showed the similar phenotypes of hypocotyl length to max2, which indicated that MAX2 is epistatic to PIF (Shen et al., 2012). But the specific relationship between PIF1 and MAX2 in the process of seedling establishment is still unclear. Interestingly, the de-etiolation phenotype of cop 1 could be partially suppressed by $\max 2$, while hypocotyl elongation in $\max 2$ could be suppressed by cop1. This result suggests COP1 may be parallel or epistatic to MAX2 (Shen et al., 2012). As a positive regulator of photomorphogenesis, HY5 acts downstream of multiple photoreceptors. But the hypocotyl length of hy5max2 was significantly longer than both hy5 and max2 which suggests that MAX2 regulates KARs and SL responses independently of HY5 (Waters and Smith, 2013). The evidence mentioned above indicated that MAX2 has an interaction with light signaling pathway, but the specific mechanisms still need more investigation.

In addition to the interaction of MAX2 with light signaling pathway, the relationship between MAX2 and phytohormones has also been investigated. IAA up-regulated the expression of SL biosynthesis genes, and the latter repressed the transportation of IAA in a MAX2-dependent manner (Foo et al., 2005; Hayward et al., 2009). This evidence suggests that MAX2 may be involved in regulating IAA transportation. Subsequent studies also showed that MAX2 can suppress the IAA transport by inhibiting the transcription of PIN genes which regulate IAA transportation (Bennett et al., 2006; Nodzynski et al., 2016). Consequently, increased IAA transportation in $\max 2$ contributes to the long hypocotyl phenotype (Shen et al., 2012). Furthermore, MAX2 also regulated the biosynthesis of $\mathrm{ABA}$ and $\mathrm{GA}$ to affect seed germination positively (Shen et al., 2012). Based on the evidence described above, MAX2 is involved in the crosstalk of phytohormones to regulate seed germination and photomorphogenesis.

KARRIKIN INSENSITIVE2 was initially named as HYPOSENSITIVE TO LIGHT (HTL) (Sun and Ni, 2011). Like max2, kai2 mutants also showed a long hypocotyl phenotype (Waters et al., 2012). Interestingly, the double mutant hy5kai2 showed longer hypocotyl compared to hy5 and kai2, which is similar to that of hy5max2 (Waters and Smith, 2013). It indicates that KAI2 regulates seedling establishment independently of HY5. But the relationship of KAI2 with other photomorphogenesis regulating factors such as PIFs and COP1 still needs more research.

\section{CONCLUSION AND FUTURE PERSPECTIVES}

The studies discussed above show that KARs can regulate seed germination and seedling development by regulating the crosstalk among endogenous phytohormones such as ABA, GA, and IAA. For intensively understanding the relationship among KARs and these phytohormones, there are still remaining important questions to be dissected.

Although ABA and IAA can induce seed dormancy synergistically while GA and KARs can accelerate germination, there is still no direct evidence that shows that KARs can affect the content or signaling pathway of IAA during the process of seed germination. The specific mechanism underlying KARs regulating endogenous phytohormones during seed germination is still unclear, especially for IAA. Furthermore, KARs could promote seedling development of Arabidopsis and inhibit the 
expression of IAA1 (Nelson et al., 2011). Thus, whether this promotion effect is due to the suppression effect of KARs on the IAA signaling pathway still needs further investigation. This hypothesis will be valuable for modern agriculture systems which suffers yield loss from shade avoidance response.

In addition to phytohormones, ROS is also involved in regulating seed dormancy and germination. The ability to interact with lipids, DNA and protein molecules in the cell makes ROS an important regulator during seed germination (Oracz et al., 2007; Oracz and Karpiński, 2016). Since ROS continuously exists in the processes of seed development stages and during storage (Pukacka and Ratajczak, 2005; Bailly et al., 2008; Leymarie et al., 2012), the phytohormones such as ABA and GA have been demonstrated to interact with ROS in regulating seed germination (Oracz and Karpiński, 2016; Shu et al., 2016). Therefore, whether KARs can regulate seed germination by interacting with ROS mediated by ABA or GA is an interesting hypothesis.

In terms of regulating seed germination, the distinct species originated from different areas might lead to different responsiveness to KARs. However, whether KARs have the similar or distinct effects on wild cultivars and cultivated cultivars within one species still needs more investigation, especially in crops species including wheat, maize and rice.

Finally, it is noted that kai2 shows a similar hypocotyl elongation phenotype to max2, which has increased IAA transportation (Waters et al., 2012). Whether there is an increase of IAA transportation in kai2 is still unknown. Consequently, whether KAI2 is also involved in the IAA signaling pathway just like MAX2 needs more investigation. Further, as positive photomorphogenesis regulators, both KAI2 and

\section{REFERENCES}

Ali-Rachedi, S., Bouinot, D., Wagner, M. H., Bonnet, M., Sotta, B., Grappin, P., et al. (2004). Changes in endogenous abscisic acid levels during dormancy release and maintenance of mature seeds: studies with the Cape Verde Islands ecotype, the dormant model of Arabidopsis thaliana. Planta 219, 479-488. doi: 10.1007/s00425-004-1251-4

Bailly, C., El-Maarouf-Bouteau, H., and Corbineau, F. (2008). From intracellular signaling networks to cell death: the dual role of reactive oxygen species in seed physiology. C. R. Biol. 331, 806-814. doi: 10.1016/j.crvi.2008.07.022

Ballaré, C. L., Mazza, C. A., Austin, A. T., and Pierik, R. (2012). Canopy light and plant health. Plant Physiol. 160, 145-155. doi: 10.1104/pp.112.200733

Bennett, T., and Leyser, O. (2014). Strigolactone signalling: standing on the shoulders of DWARFs. Curr. Opin. Plant Biol. 22, 7-13. doi: 10.1016/j.pbi.2014. 08.001

Bennett, T., Sieberer, T., Willett, B., Booker, J., Luschnig, C., and Leyser, O. (2006). The Arabidopsis MAX pathway controls shoot branching by regulating auxin transport. Curr. Biol. 16, 553-563. doi: 10.1016/j.cub.2006.01.058

Bernardo-García, S., de Lucas, M., Martínez, C., Espinosa-Ruiz, A., Davière, J.-M., and Prat, S. (2014). BR-dependent phosphorylation modulates PIF4 transcriptional activity and shapes diurnal hypocotyl growth. Genes Dev. 28, 1681-1694. doi: 10.1101/gad.243675.114

Bethke, P. C., Libourel, I. G., and Jones, R. L. (2006). Nitric oxide reduces seed dormancy in Arabidopsis. J. Exp. Bot. 57, 517-526. doi: 10.1093/jxb/erj060

Boyer, F. D., de Saint Germain, A., Pillot, J. P., Pouvreau, J. B., Chen, V. X., Ramos, S., et al. (2012). Structure-activity relationship studies of strigolactonerelated molecules for branching inhibition in garden pea: molecule design for shoot branching. Plant Physiol. 159, 1524-1544. doi: 10.1104/pp.112.195826
MAX2 show an interaction with the light signaling pathway which is incompletely understood. Furthermore, recent studies showed that KAI2 may perceive non-KARs signals (Conn and Nelson, 2015; Waters et al., 2015).Therefore, the impaired photomorphogenesis phenotypes of kai2 and max2 suggests a possible signaling pathway which is independent of KARs, but KARs can enhance the signaling outputs by interacting with the signaling networks of different phytohormones.

\section{AUTHOR CONTRIBUTIONS}

Conceived and designed the manuscript: KS and WY. Analyzed KAR and SL relationship: KS, YM, HS, and XL. Analyzed KAR regulates seed germination: YM, HS, FC, and WZ. Analyzed KAR involved in shade response: KS, FC, and WZ.

\section{FUNDING}

This work was supported by China Postdoctoral Science Foundation funded project (2014M552377 and 2016T90868), Funding of Department of Education Sichuan Province (16ZB0040) and the Natural Science Foundation of China (31071373).

\section{ACKNOWLEDGMENT}

We apologize to the colleagues whose work could not be discussed because of space limitations.

Casal, J. J. (2012). Shade avoidance. Arabidopsis Book 10:e0157. doi: 10.1199/tab. 0157

Casal, J. J. (2013a). Canopy light signals and crop yield in sickness and in health. ISRN Agron. 2013:650439. doi: 10.1155/2013/650439

Casal, J. J. (2013b). Photoreceptor signaling networks in plant responses to shade. Annu. Rev. Plant Biol. 64, 403-427. doi: 10.1146/annurev-arplant-050312120221

Chapman, E. J., Greenham, K., Castillejo, C., Sartor, R., Bialy, A., Sun, T.-P., et al. (2012). Hypocotyl transcriptome reveals auxin regulation of growth-promoting genes through GA-dependent and-independent pathways. PLoS ONE 7:e36210. doi: 10.1371/journal.pone.0036210

Chen, I. J., Lo, W. S., Chuang, J. Y., Cheuh, C. M., Fan, Y. S., Lin, L. C., et al. (2013). A chemical genetics approach reveals a role of brassinolide and cellulose synthase in hypocotyl elongation of etiolated Arabidopsis seedlings. Plant Sci. 209, 46-57. doi: 10.1016/j.plantsci.2013.04.005

Cheng, Y., Dai, X., and Zhao, Y. (2006). Auxin biosynthesis by the YUCCA flavin monooxygenases controls the formation of floral organs and vascular tissues in Arabidopsis. Genes Dev. 20, 1790-1799. doi: 10.1101/gad.1415106

Cole, B., Kay, S. A., and Chory, J. (2011). Automated analysis of hypocotyl growth dynamics during shade avoidance in Arabidopsis. Plant J. 65, 991-1000. doi: 10.1111/j.1365-313X.2010.04476.x

Conn, C. E., and Nelson, D. C. (2015). Evidence that KARRIKIN-INSENSITIVE2 (KAI2) receptors may perceive an unknown signal that is not karrikin or strigolactone. Front. Plant Sci. 6:1219. doi: 10.3389/fpls.2015.01219

Daws, M. I., Davies, J., Pritchard, H. W., Brown, N. A., and Van Staden, J. (2007). Butenolide from plant-derived smoke enhances germination and seedling growth of arable weed species. Plant Growth Regul. 51, 73-82. doi: 10.1007/ s10725-006-9149-8 
Dixon, K. W., Merritt, D. J., Flematti, G. R., and Ghisalberti, E. L. (2009). Karrikinolide: a phytoreactive compound derived from smoke with applications in horticulture, ecological restoration, and agriculture. Acta Hortic. 813, $155-170$.

Dor, E., Yoneyama, K., Wininger, S., Kapulnik, Y., Yoneyama, K., Koltai, H., et al. (2010). Strigolactone deficiency confers resistance in tomato line SL-ORT1 to the parasitic weeds Phelipanche and Orobanche spp. Phytopathology 101, 213-222. doi: 10.1094/PHYTO-07-10-0184

Drewes, F., Smith, M., and Van Staden, J. (1995). The effect of a plant-derived smoke extract on the germination of light-sensitive lettuce seed. Plant Growth Regul. 16, 205-209. doi: 10.1007/BF00029542

Eastmond, P. J., Astley, H. M., Parsley, K., Aubry, S., Williams, B. P., Menard, G. N., et al. (2015). Arabidopsis uses two gluconeogenic gateways for organic acids to fuel seedling establishment. Nat. Commun. 6:6659. doi: 10.1038/ncomms7659

Finch-Savage, W. E., Clay, H. A., Lynn, J. R., and Morris, K. (2010). Towards a genetic understanding of seed vigour in small-seeded crops using natural variation in Brassica oleracea. Plant Sci. 179, 582-589. doi: 10.1016/j.plantsci. 2010.06.005

Finch-Savage, W. E., and Leubner-Metzger, G. (2006). Seed dormancy and the control of germination. New Phytol. 171, 501-523. doi: 10.1111/j.1469-8137. 2006.01787.x

Finkelstein, R., Reeves, W., Ariizumi, T., and Steber, C. (2008). Molecular aspects of seed dormancy. Annu. Rev. Plant Biol. 59, 387-415. doi: 10.1146/annurev. arplant.59.032607.092740

Flematti, G. R., Dixon, K. W., and Smith, S. M. (2015). What are karrikins and how were they 'discovered'by plants? BMC Biol. 13:1. doi: 10.1186/s12915-0150219-0

Flematti, G. R., Ghisalberti, E. L., Dixon, K. W., and Trengove, R. D. (2004). A compound from smoke that promotes seed germination. Science 305, 977. doi: $10.1126 /$ science.1099944

Flematti, G. R., Ghisalberti, E. L., Dixon, K. W., and Trengove, R. D. (2009). Identification of alkyl substituted $2 \mathrm{H}$-furo [2, 3-c] pyran-2-ones as germination stimulants present in smoke. J. Agric. Food Chem. 57, 9475-9480. doi: 10.1021/ jf9028128

Flematti, G. R., Goddard-Borger, E. D., Merritt, D. J., Ghisalberti, E. L., Dixon, K. W., and Trengove, R. D. (2007). Preparation of 2H-Furo[2,3-c]pyran-2-one derivatives and evaluation of their germination-promoting activity. J. Agric. Food Chem. 55, 2189-2194. doi: 10.1021/jf0633241

Foo, E., Bullier, E., Goussot, M., Foucher, F., Rameau, C., and Beveridge, C. A. (2005). The branching gene RAMOSUS1 mediates interactions among two novel signals and auxin in pea. Plant Cell 17, 464-474. doi: 10.1105/tpc.104. 026716

Franklin, K. A. (2008). Shade avoidance. New Phytol. 179, 930-944. doi: 10.1111/j. 1469-8137.2008.02507.x

Fukui, K., Ito, S., Ueno, K., Yamaguchi, S., Kyozuka, J., and Asami, T. (2011). New branching inhibitors and their potential as strigolactone mimics in rice. Bioorg. Med. Chem. Lett. 21, 4905-4908. doi: 10.1016/j.bmcl.2011.06.019

Gilkerson, J., Kelley, D. R., Tam, R., Estelle, M., and Callis, J. (2015). Lysine residues are not required for proteasome-mediated proteolysis of the Auxin/indole acidic acid protein IAA1. Plant Physiol. 168, 708-720. doi: 10.1104/pp.15.00402

Gommers, C. M., Visser, E. J., St Onge, K. R., Voesenek, L. A., and Pierik, R. (2013). Shade tolerance: when growing tall is not an option. Trends Plant Sci. 18, 65-71. doi: org/10.1016/j.tplants.2012.09.008

Guo, Y., Zheng, Z., La Clair, J. J., Chory, J., and Noel, J. P. (2013). Smoke-derived karrikin perception by the $\alpha / \beta$-hydrolase KAI2 from Arabidopsis. Proc. Natl. Acad. Sci. U.S.A. 110, 8284-8289. doi: 10.1073/pnas. 1306265110

Hayward, A., Stirnberg, P., Beveridge, C., and Leyser, O. (2009). Interactions between auxin and strigolactone in shoot branching control. Plant Physiol. 151, 400-412. doi: 10.1104/pp.109.137646

Imran, M., Mahmood, A., Römheld, V., and Neumann, G. (2013). Nutrient seed priming improves seedling development of maize exposed to low root zone temperatures during early growth. Eur. J. Agron. 49, 141-148. doi: 10.1016/j. eja.2013.04.001

Jain, N., Kulkarni, M. G., and van Staden, J. (2006). A butenolide, isolated from smoke, can overcome the detrimental effects of extreme temperatures during tomato seed germination. Plant Growth Regul. 49, 263-267. doi: 10.1007/ s10725-006-9136-0
Jia, K.-P., Luo, Q., He, S.-B., Lu, X.-D., and Yang, H.-Q. (2014). Strigolactoneregulated hypocotyl elongation is dependent on cryptochrome and phytochrome signaling pathways in Arabidopsis. Mol. Plant 7, 528-540. doi: $10.1093 / \mathrm{mp} / \mathrm{sst} 093$

Jiang, L., Liu, X., Xiong, G., Liu, H., Chen, F., Wang, L., et al. (2013). DWARF 53 acts as a repressor of strigolactone signalling in rice. Nature 504, 401-405. doi: $10.1038 /$ nature 12870

Kagiyama, M., Hirano, Y., Mori, T., Kim, S. Y., Kyozuka, J., Seto, Y., et al. (2013). Structures of D14 and D14L in the strigolactone and karrikin signaling pathways. Genes Cells 18, 147-160. doi: 10.1111/gtc.12025

Keeley, S. C., and Pizzorno, M. (1986). Charred wood stimulated germination of two fire-following herbs of the California chaparral and the role of hemicellulose. Am. J. Bot. 73, 1289-1297. doi: 10.2307/2444063

Keuskamp, D. H., Pollmann, S., Voesenek, L. A. C. J., Peeters, A. J. M., and Pierik, R. (2010). Auxin transport through PIN-FORMED 3 (PIN3) controls shade avoidance and fitness during competition. Proc. Natl. Acad. Sci. U.S.A. 107, 22740-22744. doi: 10.1073/pnas.1013457108

Kobata, T., Sugawara, M., and Takatu, S. (2000). Shading during the early grain filling period does not affect potential grain dry matter increase in rice. Agron. J. 92, 411-417. doi: 10.2134/agronj2000.923411x

Leymarie, J., Vitkauskaité, G., Hoang, H. H., Gendreau, E., Chazoule, V., Meimoun, P., et al. (2012). Role of reactive oxygen species in the regulation of Arabidopsis seed dormancy. Plant Cell Physiol. 53, 96-106. doi: 10.1093/pcp/ pcr129

Lilley, J. L., Gee, C. W., Sairanen, I., Ljung, K., and Nemhauser, J. L. (2012). An endogenous carbon-sensing pathway triggers increased auxin flux and hypocotyl elongation. Plant Physiol. 160, 2261-2270. doi: 10.1104/pp.112. 205575

Liu, X., Zhang, H., Zhao, Y., Feng, Z., Li, Q., Yang, H.-Q., et al. (2013). Auxin controls seed dormancy through stimulation of abscisic acid signaling by inducing ARF-mediated ABI3 activation in Arabidopsis. Proc. Natl. Acad. Sci. U.S.A. 110, 15485-15490. doi: 10.1073/pnas.1304651110

Meng, Y., Chen, F., Shuai, H., Luo, X., Ding, J., Tang, S., et al. (2016). Karrikins delay soybean seed germination by mediating abscisic acid and gibberellin biogenesis under shaded conditions. Sci. Rep. 6:22073. doi: 10.1038/srep22073

Morffy, N., Faure, L., and Nelson, D. C. (2016). Smoke and hormone mirrors: action and evolution of karrikin and strigolactone signaling. Trends Genet. 32, 176-188. doi: 10.1016/j.tig.2016.01.002

Nagpal, P., Walker, L. M., Young, J. C., Sonawala, A., Timpte, C., Estelle, M., et al. (2000). AXR2 encodes a member of the Aux/IAA protein family. Plant Physiol. 123, 563-574. doi: 10.1104/pp.123.2.563

Nelson, D. C., Flematti, G. R., Riseborough, J. A., Ghisalberti, E. L., Dixon, K. W., and Smith, S. M. (2010). Karrikins enhance light responses during germination and seedling development in Arabidopsis thaliana. Proc. Natl. Acad. Sci. U.S.A. 107, 7095-7100. doi: 10.1073/pnas.0911635107

Nelson, D. C., Riseborough, J. A., Flematti, G. R., Stevens, J., Ghisalberti, E. L., Dixon, K. W., et al. (2009). Karrikins discovered in smoke trigger Arabidopsis seed germination by a mechanism requiring gibberellic acid synthesis and light. Plant Physiol. 149, 863-873. doi: 10.1104/pp.108.131516

Nelson, D. C., Scaffidi, A., Dun, E. A., Waters, M. T., Flematti, G. R., Dixon, K. W., et al. (2011). F-box protein MAX2 has dual roles in karrikin and strigolactone signaling in Arabidopsis thaliana. Proc. Natl. Acad. Sci. U.S.A. 108, 8897-8902. doi: 10.1073/pnas.1100987108

Nodzynski, T., Vanneste, S., Zwiewka, M., Pernisova, M., Hejatko, J., and Friml, J. (2016). Enquiry into the topology of plasma membrane localized PIN auxin transport components. Mol. Plant 9, 1504-1519. doi: 10.1016/j.molp.2016. 08.010

Oh, E., Zhu, J.-Y., Bai, M.-Y., Arenhart, R. A., Sun, Y., and Wang, Z.-Y. (2014). Cell elongation is regulated through a central circuit of interacting transcription factors in the Arabidopsis hypocotyl. Elife 3:e03031. doi: 10.7554/eLife.03031

Oracz, K., El-Maarouf Bouteau, H., Farrant, J. M., Cooper, K., Belghazi, M., Job, C., et al. (2007). ROS production and protein oxidation as a novel mechanism for seed dormancy alleviation. Plant J. 50, 452-465. doi: 10.1111/j.1365-313X.2007. 03063.x

Oracz, K., El-Maarouf-Bouteau, H., Kranner, I., Bogatek, R., Corbineau, F., and Bailly, C. (2009). The mechanisms involved in seed dormancy alleviation by hydrogen cyanide unravel the role of reactive oxygen species as key factors of 
cellular signaling during germination. Plant Physiol. 150, 494-505. doi: 10.1104/ pp.109.138107

Oracz, K., and Karpiński, S. (2016). Phytohormones signaling pathways and ROS involvement in seed germination. Front. Plant Sci. 7:864. doi: 10.3389/fpls.2016. 00864

Oracz, K., and Stawska, M. (2016). Cellular recycling of proteins in seed dormancy alleviation and germination. Front. Plant Sci. 7:1128. doi: 10.3389/fpls.2016. 01128

Petrasek, J., Mravec, J., Bouchard, R., Blakeslee, J. J., Abas, M., Seifertova, D., et al. (2006). PIN proteins perform a rate-limiting function in cellular auxin efflux. Science 312, 914-918. doi: 10.1126/science.1123542

Procko, C., Crenshaw, C. M., Ljung, K., Noel, J. P., and Chory, J. (2014). Cotyledongenerated auxin is required for shade-induced hypocotyl growth in Brassica rapa. Plant Physiol. 165, 1285-1301. doi: 10.1104/pp.114.241844

Pukacka, S., and Ratajczak, E. (2005). Production and scavenging of reactive oxygen species in Fagus sylvatica seeds during storage at varied temperature and humidity. J. Plant Physiol. 162, 873-885. doi: 10.1016/j.jplph.2004.10.012

Quinhone, A., and Ida, E. (2015). Profile of the contents of different forms of soybean isoflavones and the effect of germination time on these compounds and the physical parameters in soybean sprouts. Food chem. 166, 173-178. doi: 10.1016/j.foodchem.2014.06.012

Ramaih, S., Guedira, M., and Paulsen, G. M. (2003). Relationship of indoleacetic acid and tryptophan to dormancy and preharvest sprouting of wheat. Funct. Plant Biol. 30, 939-945. doi: 10.1071/FP03113

Sabatini, S., Beis, D., Wolkenfelt, H., Murfett, J., Guilfoyle, T., Malamy, J., et al. (1999). An auxin-dependent distal organizer of pattern and polarity in the Arabidopsis root. Cell 99, 463-472. doi: 10.1016/S0092-8674(00)81535-4

Scaffidi, A., Waters, M. T., Ghisalberti, E. L., Dixon, K. W., Flematti, G. R., and Smith, S. M. (2013). Carlactone-independent seedling morphogenesis in Arabidopsis. Plant J. 76, 1-9. doi: 10.1111/tpj.12265

Schwechheimer, C. (2008). Understanding gibberellic acid signaling-are we there yet? Curr. Opin. Plant Biol. 11, 9-15. doi: 10.1016/j.pbi.2007.10.011

Seo, M., Hanada, A., Kuwahara, A., Endo, A., Okamoto, M., Yamauchi, Y., et al. (2006). Regulation of hormone metabolism in Arabidopsis seeds: phytochrome regulation of abscisic acid metabolism and abscisic acid regulation of gibberellin metabolism. Plant J. 48, 354-366. doi: 10.1111/j.1365-313X.2006.02881.x

Shen, H., Luong, P., and Huq, E. (2007). The F-box protein MAX2 functions as a positive regulator of photomorphogenesis in Arabidopsis. Plant Physiol. 145, 1471-1483. doi: 10.1104/pp.107.107227

Shen, H., Zhu, L., Bu, Q. Y., and Huq, E. (2012). MAX2 affects multiple hormones to promote photomorphogenesis. Mol. Plant 5, 750-762. doi: 10.1093/mp/ sss029

Shin, J., Kim, K., Kang, H., Zulfugarov, I. S., Bae, G., Lee, C.-H., et al. (2009). Phytochromes promote seedling light responses by inhibiting four negativelyacting phytochrome-interacting factors. Proc. Natl. Acad. Sci. U.S.A. 106, 7660-7665. doi: 10.1073/pnas.0812219106

Shinohara, N., Taylor, C., and Leyser, O. (2013). Strigolactone can promote or inhibit shoot branching by triggering rapid depletion of the auxin efflux protein PIN1 from the plasma membrane. PLoS Biol. 11:e1001474. doi: 10.1371/journal. pbio. 1001474

Shu, K., Liu, X.-D., Xie, Q., and He, Z.-H. (2016). Two faces of one seed: hormonal regulation of dormancy and germination. Mol. Plant 9, 34-45. doi: 10.1016/j. molp.2015.08.010

Shu, K., Meng, Y., Shuai, H., Liu, W., Du, J., Liu, J., et al. (2015). Dormancy and germination: how does the crop seed decide? Plant Biol. 17, 1104-1112. doi: $10.1111 /$ plb.12356

Shu, K., Zhang, H., Wang, S., Chen, M., Wu, Y., Tang, S., et al. (2013). ABI4 regulates primary seed dormancy by regulating the biogenesis of abscisic acid and gibberellins in arabidopsis. PLoS Genet. 9:e1003577. doi: 10.1371/journal. pgen. 1003577

Smith, S. M., and Li, J. (2014). Signalling and responses to strigolactones and karrikins. Curr. Opin. Plant Biol. 21, 23-29. doi: 10.1016/j.pbi.2014.06.003

Stamm, P., and Kumar, P. P. (2010). The phytohormone signal network regulating elongation growth during shade avoidance. J. Exp. Bot. 61, 2889-2903. doi: 10.1093/jxb/erq147

Stanga, J. P., Smith, S. M., Briggs, W. R., and Nelson, D. C. (2013). SUPPRESSOR OF MORE AXILLARY GROWTH2 1 controls seed germination and seedling development in Arabidopsis. Plant Physiol. 163, 318-330. doi: 10.1104/pp.113. 221259

Stevens, J., Merritt, D., Flematti, G., Ghisalberti, E., and Dixon, K. (2007). Seed germination of agricultural weeds is promoted by the butenolide 3-methyl-2Hfuro [2, 3-c] pyran-2-one under laboratory and field conditions. Plant Soil 298, 113-124. doi: 10.1007/s11104-007-9344-Z

Stone, B. B., Stowe-Evans, E. L., Harper, R. M., Celaya, R. B., Ljung, K., Sandberg, G., et al. (2008). Disruptions in AUX1-dependent auxin influx alter hypocotyl phototropism in Arabidopsis. Mol Plant 1, 129-144. doi: 10.1093/mp/ $\operatorname{ssm} 013$

Sun, T.-P. (2011). The molecular mechanism and evolution of the GA-GID1DELLA signaling module in plants. Curr. Biol. 21, R338-R345. doi: 10.1016/ j.cub.2011.02.036

Sun, X. D., and Ni, M. (2011). HYPOSENSITIVE TO LIGHT, an alpha/beta fold protein, acts downstream of ELONGATED HYPOCOTYL 5 to regulate seedling de-etiolation. Mol. Plant 4, 116-126. doi: 10.1093/mp/ssq055

Tao, Y., Ferrer, J.-L., Ljung, K., Pojer, F., Hong, F., Long, J. A., et al. (2008). Rapid synthesis of auxin via a new tryptophan-dependent pathway is required for shade avoidance in plants. Cell 133, 164-176. doi: 10.1016/j.cell.2008. 01.049

Valladares, F., and Niinemets, $\ddot{U}$ (2008). Shade tolerance, a key plant feature of complex nature and consequences. Annu. Rev. Ecol. Evol. Syst. 39, 237-257. doi: 10.1146/annurev. ecolsys. 39. 110707. 173506

Van der Does, D., Leon-Reyes, A., Koornneef, A., Van Verk, M. C., Rodenburg, N., Pauwels, L., et al. (2013). Salicylic acid suppresses jasmonic acid signaling downstream of SCFCOI1-JAZ by targeting GCC promoter motifs via transcription factor ORA59. Plant Cell 25, 744-761. doi: 10.1105/tpc.112. 108548

Waters, M. T., Nelson, D. C., Scaffidi, A., Flematti, G. R., Sun, Y. K., Dixon, K. W., et al. (2012). Specialisation within the DWARF14 protein family confers distinct responses to karrikins and strigolactones in Arabidopsis. Development 139, 1285-1295. doi: 10.1242/dev.074567

Waters, M. T., Scaffidi, A., Flematti, G. R., and Smith, S. M. (2013). The origins and mechanisms of karrikin signalling. Curr. Opin. Plant Biol. 16, 667-673. doi: 10.1016/j.pbi.2013.07.005

Waters, M. T., Scaffidi, A., Moulin, S. L., Sun, Y. K., Flematti, G. R., and Smith, S. M. (2015). A Selaginella moellendorffii ortholog of KARRIKIN INSENSITIVE2 functions in Arabidopsis development but cannot mediate responses to karrikins or strigolactones. Plant Cell 27, 1925-1944. doi: 10.1105/ tpc. 15.00146

Waters, M. T., Scaffidi, A., Sun, Y. K., Flematti, G. R., and Smith, S. M. (2014). The karrikin response system of Arabidopsis. Plant J. 79, 623-631. doi: 10.1111/tpj. 12430

Waters, M. T., and Smith, S. M. (2013). KAI2-and MAX2-mediated responses to karrikins and strigolactones are largely independent of HY5 in Arabidopsis seedlings. Mol. Plant 6, 63-75. doi: 10.1093/mp/sss 127

Wit, M., Spoel, S. H., Sanchez-Perez, G. F., Gommers, C. M., Pieterse, C. M., Voesenek, L. A., et al. (2013). Perception of low red: far-red ratio compromises both salicylic acid-and jasmonic acid-dependent pathogen defences in Arabidopsis. Plant J. 75, 90-103. doi: 10.1111/tpj.12203

Xi, W., Liu, C., Hou, X., and Yu, H. (2010). MOTHER OF FT AND TFL1 regulates seed germination through a negative feedback loop modulating ABA signaling in Arabidopsis. Plant Cell 22, 1733-1748. doi: 10.1105/tpc.109.073072

Xu, T., Wen, M., Nagawa, S., Fu, Y., Chen, J.-G., Wu, M.-J., et al. (2010). Cell surface-and rho GTPase-based auxin signaling controls cellular interdigitation in Arabidopsis. Cell 143, 99-110. doi: 10.1016/j.cell.2010.09.003

Yamauchi, Y., Ogawa, M., Kuwahara, A., Hanada, A., Kamiya, Y., and Yamaguchi, S. (2004). Activation of gibberellin biosynthesis and response pathways by low temperature during imbibition of Arabidopsis thaliana seeds. Plant Cell 16, 367-378. doi: 10.1105/tpc.018143

Yang, X., Lee, S., So, J. H., Dharmasiri, S., Dharmasiri, N., Ge, L., et al. (2004). The IAA1 protein is encoded by AXR5 and is a substrate of SCFTIR1. Plant J. 40, 772-782. doi: 10.1111/j.1365-313X.2004.02254.x

Yoneyama, K., Yoneyama, K., Takeuchi, Y., and Sekimoto, H. (2007). Phosphorus deficiency in red clover promotes exudation of orobanchol, the signal for mycorrhizal symbionts and germination stimulant for root parasites. Planta 225, 1031-1038. doi: 10.1007/s00425-006-0410-1 
Zhao, L.-H., Zhou, X. E., Wu, Z.-S., Yi, W., Xu, Y., Li, S., et al. (2013). Crystal structures of two phytohormone signal-transducing $\alpha / \beta$ hydrolases: karrikinsignaling KAI2 and strigolactone-signaling DWARF14. Cell Res. 23, 436-439. doi: $10.1038 /$ cr.2013.19

Zhou, F., Lin, Q., Zhu, L., Ren, Y., Zhou, K., Shabek, N., et al. (2013). D14-SCFD3dependent degradation of D53 regulates strigolactone signalling. Nature 504, 406-410. doi: 10.1038/nature12878

Zhou, X.-Y., Song, L., and Xue, H.-W. (2013). Brassinosteroids regulate the differential growth of Arabidopsis hypocotyls through auxin signaling components IAA19 and ARF7. Mol. Plant 6, 887-904. doi: 10.1093/mp/ sss 123
Conflict of Interest Statement: The authors declare that the research was conducted in the absence of any commercial or financial relationships that could be construed as a potential conflict of interest.

Copyright (C) 2017 Meng, Shuai, Luo, Chen, Zhou, Yang and Shu. This is an openaccess article distributed under the terms of the Creative Commons Attribution License (CC BY). The use, distribution or reproduction in other forums is permitted, provided the original author(s) or licensor are credited and that the original publication in this journal is cited, in accordance with accepted academic practice. No use, distribution or reproduction is permitted which does not comply with these terms. 